\title{
Amyotrophic Lateral Sclerosis with Demyelinating Neuropathy
}

\author{
Haruo Nishijima ${ }^{1,2}$, Masahiko Tomiyama ${ }^{1,2}$, Chieko Suzuki ${ }^{1}$, Tomoya Kon ${ }^{1}$, \\ Yukihisa Funamizu ${ }^{1}$, Tatsuya Ueno ${ }^{1,2}$, Rie Haga ${ }^{1}$, Yasuo Miki ${ }^{1,3}$, Akira Arai ${ }^{1,2}$, \\ Tamaki Kimura ${ }^{1}$, Fumiaki Mori ${ }^{3}$, Koichi Wakabayashi ${ }^{3}$ and Masayuki Baba ${ }^{1}$
}

\begin{abstract}
Amyotrophic lateral sclerosis (ALS) with demyelinating polyneuropathy is a rare condition. We describe two ALS patients with demyelinating neuropathy. Immunomodulatory therapies brought slight symptomatic benefits to the patients, but the treatments could not halt the progression of ALS. Chance coincidence of the two diseases is unlikely in view of the low prevalence. ALS, mainly consisting of progressive axonal degeneration, might show temporal demyelinating features of peripheral nerves both electrophysiologically and pathologically. The pathomechanism for the demyelination in ALS remains to be elucidated.
\end{abstract}

Key words: amyotrophic lateral sclerosis, chronic inflammatory demyelinating polyradiculoneuropathy, electrophysiology, pathology

(Intern Med 51: 1917-1921, 2012)

(DOI: 10.2169/internalmedicine.51.7246)

\section{Introduction}

Amyotrophic lateral sclerosis (ALS) with demyelinating polyneuropathy like chronic inflammatory demyelinating polyradiculoneuropathy (CIDP) has been rarely reported (1-3). Here we describe two ALS patients with demyelinating neuropathy and demonstrate that immunomodulatory therapies are temporally beneficial for neuropathy but not for ALS.

\section{Case Reports}

\section{Case 1}

A 45-year-old Japanese right-handed man noticed numbness and then weakness and muscle atrophy in his left hand. He had no remarkable past medical history except for hypertension. When he was referred to our hospital, his cranial nerves were normal except for mild dysarthria and manual muscle testing (MMT) revealed severe weakness of the in- trinsic muscles in the left hand. Hyperreflexia was observed in the left biceps and triceps muscles, but other tendon reflexes were normal. Touch and pain sensations were mildly reduced in the distal portion of the left arm. The result of electrodiagnostic studies is shown in Table 1 (patient 1). Partial motor conduction blocks were detected outside nerve compression sites in the right ulnar nerve. Abnormal temporal dispersions were detected in the bilateral median and ulnar nerves. Needle electromyographic (EMG) examinations showed denervation and chronic neurogenic changes not only in the muscle of left upper limb but also in muscles of the tongue and all other limbs. Blood studies were normal. Anti-GM1 antibodies were not detected. Cerebrospinal fluid (CSF) was normal. No abnormalities were seen in the magnetic resonance imaging (MRI) of the brain and cervical spinal cord. Left sural nerve biopsy revealed marked onionbulb formation and scattered thin-myelinated fibers (Fig. 1a). His clinical presentations suggested ALS but nerve conduction studies and nerve biopsy findings indicated demyelinating neuropathy. His electrophysiological findings met with the electrophysiological criteria of "definite" cate-

${ }^{1}$ Department of Neurology, Aomori Prefectural Central Hospital, Japan, ${ }^{2}$ Department of Neurophysiology, Hirosaki University Graduate School of Medicine, Japan and ${ }^{3}$ Department of Neuropathology, Hirosaki University Graduate School of Medicine, Japan Received for publication January 5, 2012; Accepted for publication April 19, 2012 Correspondence to Dr. Haruo Nishijima, hnishijima-tky@umin.ac.jp 
Table 1. Results of Nerve Conduction Studies

\begin{tabular}{|c|c|c|c|c|c|}
\hline Motor Nerve & $\mathrm{DL}(\mathrm{ms})$ & $\operatorname{CMAP}(\mathrm{D} / \mathrm{P})(\mathrm{mV}) *$ & $\operatorname{MCV}(\mathrm{m} / \mathrm{s}) * *$ & F-Lat (ms). & $\mathrm{CB}$ or $\mathrm{TD}$ \\
\hline $\mathrm{RMN}$ & 4.6 & $3.6 / 2.9$ & 46 & ne & + \\
\hline RUN & 3.1 & $4.4 / \mathbf{3 . 1} / \mathbf{2 . 3}$ & $52 / 27$ & ne & + \\
\hline RTN & 4.9 & $8.5 / 4.9$ & 39 & 65 & - \\
\hline LMN & 5.9 & $1.3 / 1.3$ & 43 & 42 & + \\
\hline LUN & 5.2 & $1.1 / 0.8 / 0.8$ & $40 / 46$ & 42 & + \\
\hline LTN & 5.4 & $8.6 / 6.0$ & 41 & 41 & - \\
\hline Sensory Nerve & & SNAP $(D / P)(\mu V)$ & \multicolumn{2}{|c|}{$\operatorname{SCV}(\mathrm{D} / \mathrm{P})(\mathrm{m} / \mathrm{s})$} & \\
\hline RMN & & $17 / 7.3$ & \multicolumn{2}{|c|}{$44 / 55$} & \\
\hline RUN & & $13 / 7.1$ & \multicolumn{2}{|c|}{$40 / 42$} & \\
\hline RSN & & $6.2 / \mathrm{nd}$ & \multicolumn{2}{|c|}{$27 / \mathrm{nd}$} & \\
\hline $\mathrm{LMN}$ & & $12 / 6.7$ & \multicolumn{2}{|c|}{$40 / 45$} & \\
\hline LUN & & $7.7 / 2.8$ & \multicolumn{2}{|c|}{$34 / 41$} & \\
\hline LSN & & $8.6 / \mathrm{nd}$ & \multicolumn{2}{|c|}{$35 / \mathrm{nd}$} & \\
\hline
\end{tabular}

Patient 2

\begin{tabular}{|c|c|c|c|c|c|}
\hline Motor Nerve & DL (ms) & $\mathrm{CMAP}(\mathrm{D} / \mathrm{P})(\mathrm{mV}) *$ & $\mathrm{MCV}(\mathrm{m} / \mathrm{s})$ & F-Lat (ms) & $\mathrm{CB}$ or $\mathrm{TD}$ \\
\hline RMN & 4.2 & $6.2 / 5.7$ & 61 & 30 & - \\
\hline RUN & 5.0 & $0.8 / 0.8 / 0.7$ & 38 & 22 & + \\
\hline RTN & 5.0 & $6.7 / 4.2$ & 44 & ne & - \\
\hline LMN & 4.9 & $4.1 / 3.6$ & 51 & ne & - \\
\hline LUN & 3.9 & $1.3 / 1.0 / 0.9$ & 68 & ne & + \\
\hline LTN & 5.2 & $4.3 / 3.3$ & 37 & 53 & - \\
\hline Sensory Nerve & & SNAP $(D / P)(\mu V)$ & \multicolumn{2}{|c|}{$\mathrm{SCV}(\mathrm{D} / \mathrm{P})(\mathrm{m} / \mathrm{s})$} & \\
\hline RMN & & $15 / 9.6$ & \multicolumn{2}{|c|}{$44 / 55$} & \\
\hline RUN & & $3.3 /$ ne & \multicolumn{2}{|c|}{$39 /$ ne } & \\
\hline RSN & & $5.5 / \mathrm{nd}$ & \multicolumn{2}{|c|}{$36 /$ nd } & \\
\hline LMN & & $15 / 10$ & \multicolumn{2}{|c|}{$47 / 55$} & \\
\hline LUN & & $5.7 / 2.1$ & \multicolumn{2}{|c|}{$46 / 52$} & \\
\hline LSN & & $3.3 / \mathrm{nd}$ & \multicolumn{2}{|c|}{$32 / \mathrm{nd}$} & \\
\hline
\end{tabular}

* CMAP values in ulnar nerves of patients 1 and 2 indicate the amplitudes by distal / below elbow / above elbow stimulation. ** MCV values in ulnar nerves of patient 1 indicate conduction velocity between wrist and below elbow / below and above elbow.

Abnormal values are in bold.

Abbreviations: R: right, L: left, D: distal, P: proximal (below elbow in ulnar nerves), MN: median nerve, UN: ulnar nerve, TN: tibial nerve, SN: sural nerve, DL: distal latency, CMAP: compound muscle action potential, MCV: motor nerve conduction velocity, F-Lat.: F wave latency, SNAP: sensory nerve action potential, SCV: sensory nerve conduction velocity, CB: conduction block, TD: abnormal temporal dispersion, ne: not evoked, nd: not done

Normal values: MN DL <4.2; MN CMAP >3.5; MN MCV >48; MN F-Lat. <31; UN DL <3.4; UN CMAP >2.7; UN MCV >49; UN F-Lat. <32; TN DL <6.0; TN CMAP > 2.9; TN MCV >41; TN F-Lat. <58; MN SNAP (D/P) >19/>16; MN SCV (D/P) >44 / >53; UN SNAP (D/P) >18 / >15; UN SCV $(\mathrm{D} / \mathrm{P})>47$ / >54; $\mathrm{SN}$ SNAP $>3.8 ;$ SN SCV $>39$

gory for CIDP (European Federation of Neurological Societies/Peripheral Nerve Society (EFNS/PNS) criteria), for abnormal temporal dispersions were detected in more than two nerves (4). Intravenous immunoglobulin (IVIg) was given and hypesthesia in the left hand improved but weakness pro- gressed. In the following 10 months, weakness and muscle atrophy developed in all limbs and bulbar palsy and respiratory failure appeared. Tendon reflexes were brisk in all limbs. Fasciculation was observed in tongue and four limb muscles. The second course of IVIg, oral prednisolone, and 


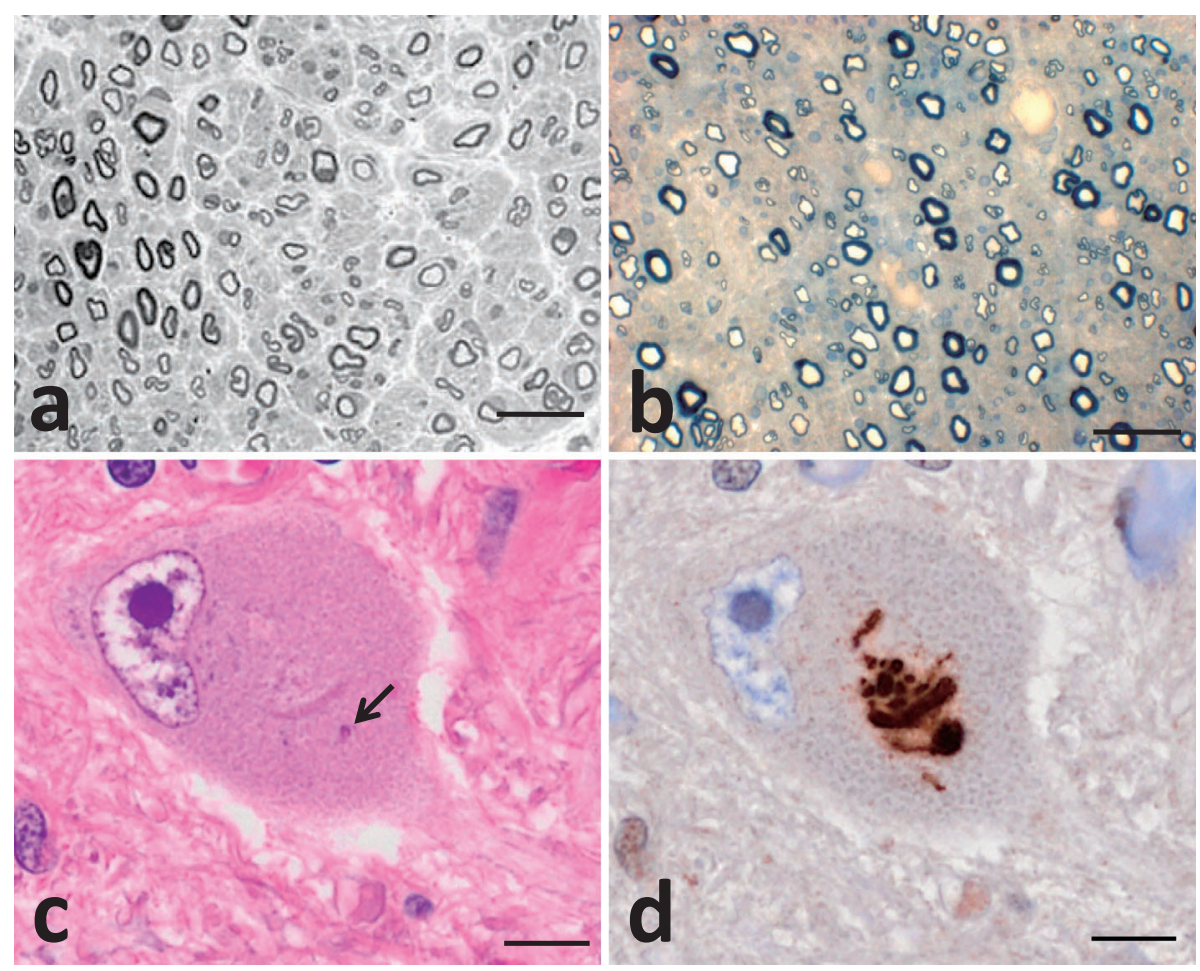

Figure 1. Pathological findings. a: Left sural nerve of patient 1 . Large myelinated fibers were decreased. There were many thinly myelinated fibers. Onion bulb formations were seen, indicating demyelinating and remyelinating processes. Lymphocytes or macrophage infiltration were not seen. Toluidine blue staining. Scale bar: $20 \mu \mathrm{m}$. b: Right sural nerve of patient 2 . Large myelinated fibers were slightly decreased.There were many thinly myelinated fibers. Lymphocytes or macrophage infiltration were not seen. Toluidine blue staining. Scale bar: $20 \mu \mathrm{m}$. c: Anterior horn cell of the spinal cord of patient 2. Bunina bodies were present in the anterior horn cells (arrow). Hematoxylin and Eosin staining. Scale bar: $10 \mu \mathrm{m}$. d: Anterior horn cell of the spinal cord of patient 2. TDP-43-immmunoreactive skein-like inclusions were seen in the anterior horn cells. Phosphorylated TDP-43 immunostaining. Scale bar: $10 \mu \mathrm{m}$.

10 plasma exchanges were without benefits. He was diagnosed with "clinically definite" ALS according to the revised El Escorial criteria (5). Four years after the onset he became bedridden and had to be artificially-ventilated.

\section{Case 2}

A 68-year-old Japanese right-handed man was admitted with a 7-month history of progressive weakness and muscle atrophy of limbs. He first noticed weakness in the left leg and then in the right arm. Six months after the onset, he developed pneumonia. He had a history of hypertension, diabetes mellitus and hypercholesterolemia. When he was referred to us, he was bedridden with dyspnea and quadriplegia. Cranial nerves were normal. MMT revealed proximally dominant symmetrical weakness and muscle atrophy of four limbs. Fasciculation was seen in the four limb muscles. Tendon reflexes were brisk in the left biceps brachii and both quadriceps femoris, but reduced in the right biceps brachii, absent in both triceps brachii and Achilles' tendons. Sensations were normal in all modalities. The results of electrodiagnostic studies are shown in Table 1 (patient 2). Negative phase durations of ulnar compound muscle action potentials by proximal stimulation were prolonged to more than $20 \mathrm{~ms}$ while those by distal stimulation were 5 to $6 \mathrm{~ms}$ in both sides. Inching stimulation techniques of forearm ulnar nerves revealed multifocal waveform changes indicating primary multifocal demyelination in both sides (Fig. 2). Needle EMG examinations suggested chronic neurogenic changes in the four limb muscles without signs of active denervation. Blood studies were normal except reduced $\mathrm{Na}^{+}$level (122 $\mathrm{mEq} / \mathrm{L})$, elevated C-reactive protein $(9.6 \mathrm{mg} / \mathrm{dL})$ and white blood cell count $\left(17,500 / \mathrm{mm}^{3}\right)$, elevated HbA1c $(6.1 \%)$ and total cholesterol $(240 \mathrm{mg} / \mathrm{dL})$. Anti-GM1 antibodies and anti-GalNac-GD1a antibodies were negative. CSF examination revealed elevated protein level of $89 \mathrm{mg} / \mathrm{dL}$, without pleocytosis. Brain and cervical spine MRI showed no remarkable abnormalities. Right sural nerve biopsy showed slightly reduced number of large myelinated fibers with many thinly myelinated fibers, suggesting demyelination (Fig. 1b). His symptoms met the diagnostic criteria of "clinically definite" ALS (5). He was also diagnosed as "definite" CIDP according to the EFNS/PNS criteria, because abnormal temporal dispersions were detected in two nerves (4). A course of IVIg was given and followed by oral prednisolone administration. One month after the treatment, muscle strength improved so that he could walk with a cane 


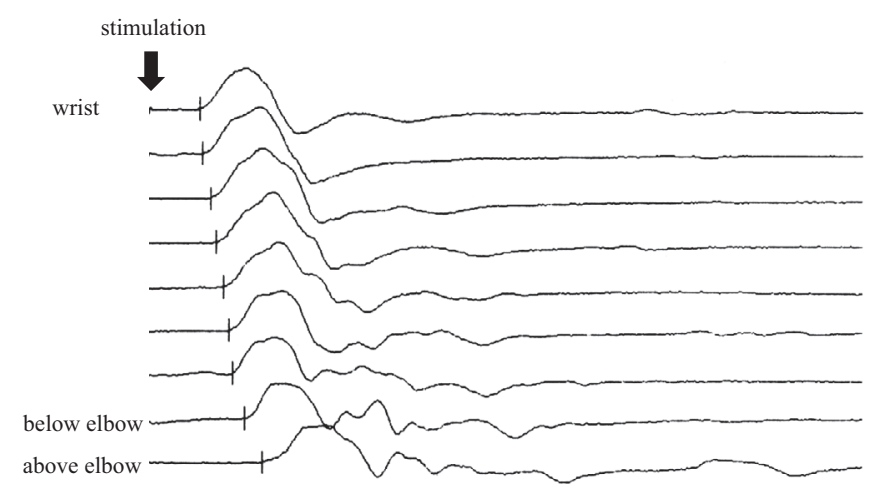

Figure 2. Inching study in the left ulnar nerve of patient 2. Multifocal waveform changes were detected.

and he was discharged. However, two months later he had restrictive ventilatory failure and was hospitalized again. His muscle weakness and atrophy progressed and he was bedridden. The second course of IVIg and two plasma exchanges were without effect. The patient suddenly died during plasma exchange. Autopsy revealed the cause of death to be acute myocardial infarction. Neuropathologic examinations demonstrated that there were multiple foci of loss of large myelinated fibers in the anterior and posterior nerve roots of the thoracic and lumbar cord, suggesting CIDP-like demyelinating neuropathy. On the other hand, neurons were lost with gliosis in the spinal anterior horn. Degeneration was observed in the bilateral corticospinal tract. Bunina bodies and TDP-43-immmunoreactive skein-like inclusions were seen in the anterior horn cells (Fig. 1c, d). These pathological findings indicate ALS with demyelinating radiculopathy.

\section{Discussion}

Here, we describe two patients with ALS. From clinical, electrophysiological and pathological findings we believe that they had demyelinating neuropathy. Minor symptomatic relief was obtained by IVIg, however ALS became advanced and both patients ended up with a poor prognosis.

It might be discussed as to whether they really had demyelinating neuropathy? It has been shown that in wasted muscles electrophysiological abnormalities such as slightly decreased motor nerve conduction velocities and partial conduction block-like reduction of compound muscle action potentials can be seen with ALS $(6,7)$. These abnormalities may be detected with axonal losses. It is also reported that sensory axonal neuropathy could occur with ALS. Sensory nerve action potentials amplitude and sensory nerve conduction velocities can be decreased with ALS (8-10). Thus the electrophysiological findings of the present two patients might not be convincing enough to confirm the existence of demyelinating neuropathy. In patient 1 marked onion bulb formations were detected with sural nerve biopsy, which we believe to be the evidence of demyelinating and remyelinating processes in his peripheral nerves. In patient 2 demyeli- nation in the anterior and posterior nerve roots could be secondary de-remyelination following progressive axonal atrophy. However, the waveform changes in inching stimulation techniques of bilateral ulnar nerves seemed to be rather multifocal than continuous, suggesting demyelination that was not only due to axonal degeneration. Moreover, patient 2 was bedridden on his first admission to our hospital, and presented temporal but substantial improvement in his muscle strength up to being able to walk with a cane after IVIg treatment, which indicates the coexistence of treatable or at least remittable neuropathy and ALS. From these findings, we concluded that both of the patients had demyelinating neuropathy associated with ALS.

It is less likely that they had inherited neuropathies such as Charcot-Marie-Tooth disease because they presented with temporal dispersion and multifocal conduction blocks (11). Furthermore, the response to immunomodulatory therapies, though they were limited, supported remittable neuropathies.

The next question to consider is whether these two patients had ALS and CIDP coincidentally? The prevalence of ALS in Japan is 6.7 for every 100,000 people (12). On the other hand, the prevalence of CIDP is 1.61 for every 100,000 people (13). Accordingly, chance coincidence of ALS and CIDP would be 10.8 for every 10 billion people. We have encountered two patients with ALS and demyelinating neuropathy in the past 10 years. Since the population of our medical district is approximately one million, chance coincidence seems less likely.

To date only ten patients with ALS and demyelinating polyneuropathy have been reported (1-3). Immunomodulatory therapies were slightly efficacious in three of the ten patients, as in the current patients (1-3), however all of the patients had a poor prognosis. The interval from disease onset to respiratory failure or death in the present two patients and the ten previously reported patients was similar to that of patients with classic ALS. Motor neuron degeneration and treatable demyelinating neuropathy must have quite a different pathogenesis. At this moment we can not conclude that the present two patients had "primary" and "treatable" demyelinating neuropathy like CIDP, because there is no valid explanation of the pathomechanism of their coexistence. However, at least we can say that our two patients had remittable demyelination in their peripheral nerves.

Thus, ALS with demyelinating neuropathy is present in limited numbers. Although immunomodulatory therapy available at the moment may be temporally beneficial for the neuropathy, unfortunately it does not decrease the progression of ALS.

\section{The authors state that they have no Conflict of Interest (COI).}

\section{Acknowledgement}

We thank Susumu Kusunoki (Department of Neurology, Kinki University School of Medicine) for measuring anti-ganglioside antibodies. 


\section{References}

1. Echaniz-Laguna A, Degos B, Mohr M, Kessler R, Urban-Kraemer E, Tranchant C. A study of three patients with amyotrophic lateral sclerosis and a polyneuropathy resembling CIDP. Muscle Nerve 33: 356-362, 2006.

2. Rajabally YA, Jacob S. Chronic inflammatory demyelinating polyneuropathy-like disorder associated with amyotrophic lateral sclerosis. Muscle Nerve 38: 855-860, 2008.

3. Sawa N, Kataoka H, Sugie K, et al. Clinical analysis and outcomes of amyotrophic lateral sclerosis with demyelinating polyneuropathy. Amyotroph Lter Acler 13: 125-131, 2012.

4. Joint Task Force of the EFNS and the PNS. European Federation of Neurological Societies/Peripheral Nerve Society Guideline on management of chronic inflammatory demyelination polyradiculoneuropathy: report of a joint task force of the European Federation of Neurological Societies and the Peripheral Nerve SocietyFirst Revision. J Peripher Nerv Syst 15: 1-9, 2010 (Erratum in: J Peripher Nerv Syst 15: 373, 2010).

5. Brooks BR, Miller RG, Swash M, Munsat TL; World Federation of Neurology Research Group on Motor Neuron Diseases. El Escorial revisited: revised criteria for the diagnosis of amyotrophic lateral sclerosis. Amyotroph Lateral Scler Other Motor Neuron Disord 1: 293-299, 2000.

6. Cornblath DR, Kuncl RW, Mellits ED, et al. Nerve conduction studies in amyotrophic lateral sclerosis. Muscle Nerve 15: 1111$1115,1992$.
7. Veugelers B, Theys P, Lammens M, Van Hees J, Robberecht W. Pathological findings in a patient with amyotrophic lateral sclerosis and multifocal motor neuropathy with conduction block. J Neurol Sci 136: 64-70, 1996.

8. Pugdahl K, Fuglsang-Frederiksen A, de Carvalho M, et al. Generalised sensory system abnormalities in amyotrophic lateral sclerosis: a European multicentre study. J Neurol Neurosurg Psychiatry 78: 746-749, 2007.

9. Isaacs JD, Dean AF, Show CE, Al-Chalabi A, Mills KR, Leigh PN. Amyotrophic lateral sclerosis with sensory neuropathy: part of a multisystem disorder? J Neurol Neurosurg Psychiatry 78: 750753, 2007.

10. Hammad M, Silva A, Glass J, Sladky JT, Benatar M. Clinical, electrophysiologic, and pathologic evidence for sensory abnormalities in ALS. Neurology 69: 2236-2242, 2007.

11. Stanton M, Pannoni V, Lewis RA, et al. Dispersion of compound muscle action potential in hereditary neuropathies and chronic inflammatory demyelinating polyneuropathy. Muscle Nerve 34: 417422, 2006.

12. Statistics and Information Department, Minister's Secretariat, Japan. Report on health administration 2009. The Ministry of Health, Labor and Welfare, Tokyo, 2010.

13. Iijima M, Koike H, Hattori N, et al; Refractory Peripheral Neuropathy Study Group of Japan. Prevalence and incidence rates of chronic inflammatory demyelinating polyneuropathy in the Japanese population. J Neurol Neurosurg Psychiatry 79: 1040-1043, 2008.

(C) 2012 The Japanese Society of Internal Medicine http://www.naika.or.jp/imindex.html 